\title{
Correlation of Trigeminopontine Angle with Severity of Trigeminal Neuralgia due to Neurovascular conflict over medial aspect of nerve. Can we prognosticate the reduction in pain in patients on medical management?
}

Pundalik Umalappa Lamani

NIMS Hyderabad

Abhishek J Arora ( $\nabla$ dr.abhiarora@gmail.com )

All India Institute of Medical Sciences

Kiran Kumar Reddy Kona

NIMS Hyderabad

Jyotsna Yarlagadda

NIMS Hyderabad

Research

Keywords: Trigeminal Neuralgia, Trigeminopontine Angle, Neurovascular Conflict

Posted Date: April 30th, 2021

DOI: https://doi.org/10.21203/rs.3.rs-415743/v1

License: (9) This work is licensed under a Creative Commons Attribution 4.0 International License.

Read Full License 


\section{Abstract}

Background: Neurovascular conflicts (NVC) are one of the major causative factors in patients presenting with Trigeminal neuralgia (TN). We found great degree of variation in acuteness of medial trigeminopontine angle (mTPA), angle between medial border of trigeminal nerve and anterior border of pons in patients with TN and tried to find its correlation with pain severity due to NVC over the medial aspect of nerve (mNVC).

Aims and Objectives: To Correlate mTPA measurement with severity of trigeminal neuralgia due to mNVC. To calculate the reduction in pain in patients kept on medical management and its correlation with medial trigeminopontine angle.

Material and Methods: This was a retrospective observational study conducted between May 2018 to October 2020. A total of 41 patients presenting with Trigeminal Neuralgia and showing corresponding Neurovascular conflict were included in the study. Out of the total cases with NVC, 30 cases showed NVC over the medial surface of the nerve. All the patients were evaluated on MAGNETOM Skyra 3T MRI (Siemens). Using Two-line Cobb angle method, trigeminopontine angle was calculated. Pre-treatment pain intensity and post treatment pain relief of each patients were assessed by using numeric rating scale (NRS). NRS with numbers from 0 to 10 ('no pain' to 'worst pain imaginable'). Relevant clinical details regarding pre- and post-treatment pain score as well as treatment plan opted by patients were collected.

Results: Patients with post treatment response $\geq 50 \%$ is considered as "good response" and $<50 \%$ is considered as "poor response". In our study with trigeminopontine angle threshold of 45 degree, 7 out of $8(87.5 \%)$ patients with $>45^{0}$ mTPA showed poor response and $15 / 22(68.2 \%)$ patients $\leq 45^{0}$ showed good response to medical management for trigeminal neuralgia due to $\mathrm{mNVC}$ with statistical significance difference with $\mathrm{p}$-valve of 0.007

Conclusion: In our study, we found a negative correlation between the MTPA and percentage pain relief in patients kept on medical management. We realised that mTPA measurement could become an important tool for prognosticating pain relief for patients of trigeminal neuralgia on medical therapy.

\section{Introduction}

A wide variety of abnormalities can lead to trigeminal neuropathy, which include lesions involving the trigeminal nerve itself (primary lesions) and lesions that secondarily involve the nerve or any one of its three branches. Anatomically, trigeminal nerve is divided into 4 segments, based on its course through the brain stem, prepontine cistern, Meckel cave \& cavernous sinus, and extracranially. ${ }^{(1,2)}$

Trigeminal neuralgia (TN) is described as a sudden onset of severe, unilateral, paroxysmal, and lancinating pain in one or more of the distributions of the trigeminal nerve. Vascular compression, also known as neurovascular conflict, of the trigeminal nerve is one of the commonest secondary known 
cause, as suggested by Dandy in 1932, of TN. In his series, he reported that the superior cerebellar artery caused NVC in $30.7 \%$ of patients. $(2,5,8)$ In another series, it was found that only $0-8.5 \%$ of cases of TN, were secondary to tumours. ${ }^{(3)}$ Studies have already been published correlating compression, displacement and cross sectional area of trigeminal nerve with the severity of pain. We couldn't find any study correlating the MTPA with pain severity in review of literature. NVC of nerve could be on either side of the nerve, however, since the TPA is acute over the medial aspect, we tried to find the correlation of acuteness of mTPA with the $\mathrm{MNVC}$ pain responsiveness.

AIMS AND OBJECTIVES: To Correlate mTPA measurement with severity of trigeminal neuralgia due to $\mathrm{mNVC}$ and to evaluate the reduction in pain in patients kept on medical management and its correlation with medial trigeminopontine angle.

\section{Material And Method}

This was a retrospective observational study conducted between May 2018 to October 2020.

Study population: A total of 42 cases with suspected trigeminal neuralgias which were referred to department of Radiology for MRI scan were evaluated. Out of which a total of 30 cases of TN due to NVC on medial aspect of trigeminal nerve were found and included in our study, with age ranging between 22 to 82 years including 19 males and 11 females. An informed consent was taken from each patient for their inclusion in the study. (Figure 1)

Inclusion criteria: Cases with TN with instigating vessel abutting/compressing over medial aspect of trigeminal nerve, and who were managed conservatively on medical management.

Exclusion criteria: Patients who underwent prior surgical microvascular decompression, NVC over lateral aspect of trigeminal nerve or patients presenting with TN due to any other etiology were excluded.

Imaging protocol: All the patients were evaluated on MAGNETOM Skyra 3T MRI (Siemens). Sequences acquired for evaluation were i) T1- axial sections of whole brain; ii) T2-axial sections of whole brain and posterior fossa (medulla to upper pons) with iii) thin slices 3D T2-SPACE Sequence; and iv) FLAIR-axial sections of whole brain. Bilaterally, trigeminal nerves were examined for vascular compression/contact. In multiplanar windows, point of contact between the instigating vessel and the nerve was identified and only cases with medial point/area of contact with the nerve and vessel were included. Later, trigeminopontine angle was calculated. Relevant clinical details regarding pre- and post-treatment pain score as well as other laboratory investigations performed for the cases were collected. Microsoft excel software was used to analyse the data. Charts and tables were prepared for representation and comparison of data.

Trigeminopontine angle calculation: Using Two-line Cobb angle method, trigeminopontine angle was calculated on symptomatic side in T2 3D SPACE- axial sections of MRI brain at the level of trigeminal nerve. A line is drawn along medial aspect of cisternal segment of trigeminal nerve and second line is 
drawn along anterior aspect of pons medially, the resultant formation of angle is taken as medial trigeminopontine angle (mTPA)(Figure 2).

Pain scale: Assessment of pre-treatment pain intensity and post treatment pain relief was done using numeric rating scale (NRS). NRS with numbers from 0 to 10 ('no pain' to 'worst pain imaginable') were evaluated from all the patients at the time of MRI and at the time of follow up visit after a course of medical therapy. Pre and post treatment pain relief response score (0 to 10) were converted into percentages ( 0 to $100 \%$ ) and then the patients were divided into two groups. Patients with post treatment response of equal or more than $50 \%$, i.e., significant response were considered showing "good response" and those with less than $50 \%$ of post treatment pain relief, were considered as showing "poor response" to medical management.

Statistical Analysis: Pearson Chi-Square test showed that statistical significance difference with $p$-valve of 0.007 , between patients with post treatment pain relief good response $(\geq 50 \%)$ and poor $/ \mathrm{bad}$ response $(<50 \%)$ groups with trigeminopontine angle threshold of 45 degree. Pearson correlation coefficient test was done for two variables using post treatment pain relief in percentage and trigeminopontine angle in degree, and resultant R-value was -0.3366 , which showed negative correlation. Thus, patients with more acute mTPA showed better response to medical management.

\section{Results}

A total 30 cases of trigeminal neuralgia due to neurovascular compression over medial aspect of trigeminal nerve were evaluated. Presenting age of patients varied between 22years to 82 years, including 19 males and 11 females. Majority of our patients were within 41-50 years (30\%) and 51-60 years (23.3\%) age group.

Pre-treatment pain intensity and post treatment pain relief of each patient was assessed by using numeric rating scale (NRS). NRS with numbers from 0 to 10 ('no pain' to 'worst pain imaginable') was used in subjective assessment of pain. As described, the post treatment pain relief response score ( 0 to 10) was converted into percentages ( 0 to $100 \%$ ) and then patients divided into two groups, of those showing significant or 'good response' and those showing 'poor response'. These pain relief findings were correlated with the medial TPA. In our study, on keeping trigeminopontine angle threshold of $45^{\circ}, 7$ out of $8(87.5 \%)$ patients $>45^{0} \mathrm{mTPA}$ showed bad/poor response and $15 / 22(68.2 \%)$ patients $\leq 45^{0} \mathrm{mTPA}$ showed good response to medical management for trigeminal neuralgia due to neurovascular conflict with statistical significance difference with $p$-valve of 0.007 . Pearson correlation coefficient test was done for post treatment pain relief in percentage and mTPA, which showed resultant R-value of -0.3366 , suggesting negative correlation. (Figure 3)

\section{Discussion}


A wide variety of abnormalities can lead to trigeminal neuralgia (TN), including pathologies involving the trigeminal nerve itself (primary) and etiologies that secondarily involve the nerve or any one of its three branches. ${ }^{(1,2)}$ Neurovascular conflict is one of the commonest causes of trigeminal neuralgia involving the cisternal portion of the nerve. Other resultant pathologies causing TN include, demyelinating diseases like multiple sclerosis, vascular insults leading to infarcts, and tumours like glioma involving the trigeminal nerve at any level. Trigeminal neuropathy involving the, canalicular (Meckel cave and cavernous sinus) segment, are frequently due to meningiomas, trigeminal schwannomas, epidermoid cysts, metastases, pituitary adenomas, and aneurysms. The most common extracranial cause of TN is perineural spread of malignant tumors. ${ }^{(1,2)}$

The clinical findings do not permit accurate localization of pathologies; therefore, magnetic resonance imaging (MRI) is the modality of choice to visualize the entire course of the fifth cranial nerve and localize the pathology. ${ }^{(1,3)}$ Commonly recognized NVC syndromes encountered in clinical practice are trigeminal neuralgia, hemifacial spasm and glossopharyngeal neuralgia. The first-line treatment of patients with the aforementioned symptoms is based on conservative medical management. Patients refractory to conservative treatment later undergo microvascular decompression with good success rates. A long-term study of the results on trigeminal neuralgia after microsurgical vascular decompression (Kaplan-Meier curves at 20 years) showed that cure was achieved in $88.1 \%$ of the patients with a neurovascular compression (NVC) producing a large groove on the nerve (Grade III), $78.3 \%$ of the patients with a NVC with nerve distortion or displacement (Grade II), and $58.3 \%$ of the patients with a NVC with simple contact on the nerve (Grade I). Therefore, preoperative visualization of the NVC by MRI and determination of its grading are important for the therapeutic decision. ${ }^{(4,9)}$ There are few studies evaluating various parameters with patient outcome on medical management.

In our study, we correlated the degree of medial trigeminopontine angle with post treatment pain reduction in medically managed patients of trigeminal neuralgia due to neurovascular conflict.

The well-known visual analogue scale (VAS) and numeric rating scale (NRS) for assessment of pain intensity agree well and are equally sensitive in assessing acute pain after surgery, and they are both superior to a four-point verbal categorical rating scale (VRS). An NRS with numbers from 0 to 10 ('no pain' to 'worst pain imaginable') is more practical than a VAS, easier to understand for most people, and does not need clear vision, dexterity, paper, and pen. One can even determine the intensity of pain accurately using telephone interview, a computerized telephone interview, and recording of NRS data by the patient directly into the database of a computer via the telephone keyboard. ${ }^{(10)}$ In our study we have used numeric rating scale (NRS) method for assessment of pre-treatment pain intensity and post treatment pain relief in each patient separately. Post treatment pain relief response score ( 0 to 10$)$ was converted into percentages (0 to $100 \%$ ) and then divided into two groups as described.

In our study, Pearson correlation coefficient test was done for two variables using post treatment pain relief in percentage and trigeminopontine angle in degree, which resulted in R-value of -0.3366 , thus confirming negative correlation of MTPA with percentage of pain relief. This finding is helpful in 
prognosticating the pain relief that could be expected in patients with TN, by studying the mTPA. By keeping, trigeminopontine angle threshold of 45 degree, patients with more than $>45^{0}$ medial trigeminopontine angle showed bad/poor response, while those with less than or equal $\leq 45^{0}$ medial trigeminopontine angle showed good response to medical management for trigeminal neuralgia due to neurovascular conflict, which was statistically significant at p-valve of 0.007 ( $p<0.01)$. (Figure 4 and 5). Possible hypothesis of better medical management response in patients with lower mTPA could be attributed to wider course of the nerve, due to inherent shape of the base of the skull, however it needs further research and larger studies.

Limitations of our study were single institutional study, small sample size and subjective variation in pain relief scoring.

\section{Conclusion}

The high-resolution magnetic resonance imaging directs the physicians to decision making in the plan of action for management of trigeminal neuralgia due to neurovascular conflict. Our study is a step-in direction of prognosticating the outcome of medical management in patients presenting with TN, based on the MTPA. We found a negative correlation between the MTPA and percentage pain relief in patients kept on medical management. We realised that by keeping an angle threshold of 45 degrees, statistically significant correlation was achieved in predicting outcome of the patients. Hence, mTPA measurement could become an important tool for prognosticating pain relief for patients of trigeminal neuralgia on medical therapy.

\section{Declarations}

- Ethics approval and consent to participate : Being a retrospective study, the IEC/IRC approval was waived.

- Consent for publication : Yes

- Availability of data and materials: The datasets used and/or analysed during the current study are available from the corresponding author on reasonable request.

- Competing interests : None

- Funding : Nil

- Authors' contributions : AA - had the idea for study, collected, analysed, wrote manuscript. PL collected data, analysed, wrote manuscript. KK - helped in proof reading, collection of data, preparation of figures. JY - helped in editing and proof reading. All authors significantly contributed in data collection, analysis, manuscript preparation, proof reading. All authors read and approved the final manuscript.

- Acknowledgements : None 


\section{References}

1. Majoie CB, Verbeeten B Jr, Dol JA, Peeters FL. Trigeminal neuropathy: evaluation with MR imaging. Radiographics. 1995 Jul;15(4):795-811.

2. Ko AL, Lee A, Raslan AM, Ozpinar A, McCartney S, Burchiel KJ. Trigeminal neuralgia without neurovascular compression presents earlier than trigeminal neuralgia with neurovascular compression. Journal of neurosurgery. 2015 Dec 1;123(6):1519-27.

3. Jamjoom AB, Jamjoom ZA, Al-Fehaily M, El-Watidy S, Al-Moallem M. Trigeminal neuralgia related to cerebellopontine angle tumors. Neurosurgical review. 1996 Dec 1;19(4):237 - 41.

4. Leal PR, Froment JC, Sindou M. MRI sequences for detection of neurovascular conflicts in patients with trigeminal neuralgia and predictive value for characterization of the conflict (particularly degree of vascular compression). Neuro-Chirurgie. 2010 Feb;56(1):43-9.

5. Hughes MA, Frederickson AM, Branstetter BF, Zhu X, Sekula RF Jr. MRI of the trigeminal nerve in patients with trigeminal neuralgia secondary to vascular compression. American Journal of Roentgenology. 2016 Mar;206(3):595-600.

6. Tanrikulu L, Scholz T, Nikoubashman O, Wiesmann M, Clusmann H. Preoperative MRI in neurovascular compression syndromes and its role for microsurgical considerations. Clinical neurology and neurosurgery. 2015 Feb 1; 129:17-20.

7. Tanrikulu L, Hastreiter P, Bassemir T, Bischoff B, Buchfelder M, Dörfler A, Naraghi R. New clinical and morphologic aspects in trigeminal neuralgia. World neurosurgery. 2016 Aug 1;92:189 - 96.

8. Blitz AM, Northcutt B, Shin J, Aygun N, Herzka DA, Theodros D, Goodwin CR, Lim M, Seeburg DP. Contrast-enhanced CISS imaging for evaluation of neurovascular compression in trigeminal neuralgia: improved correlation with symptoms and prediction of surgical outcomes. American Journal of Neuroradiology. 2018 Sep 1;39(9):1724-32.

9. Hardaway FA, Holste K, Ozturk G, Pettersson D, Pollock JM, Burchiel KJ, Raslan AM. Sex-dependent posterior fossa anatomical differences in trigeminal neuralgia patients with and without neurovascular compression: a volumetric MRI age-and sex-matched case-control study. Journal of neurosurgery. 2019 Feb 1;132(2):631-8.

10. Breivik H, Borchgrevink PC, Allen SM, Rosseland LA, Romundstad L, Breivik Hals EK, Kvarstein G, Stubhaug A. Assessment of pain. BJA: British Journal of Anaesthesia. 2008 Jul 1;101(1):17-24.

\section{Figures}




\section{2}

Total cases of Trigeminal neuralgia (TN) were

evaluated

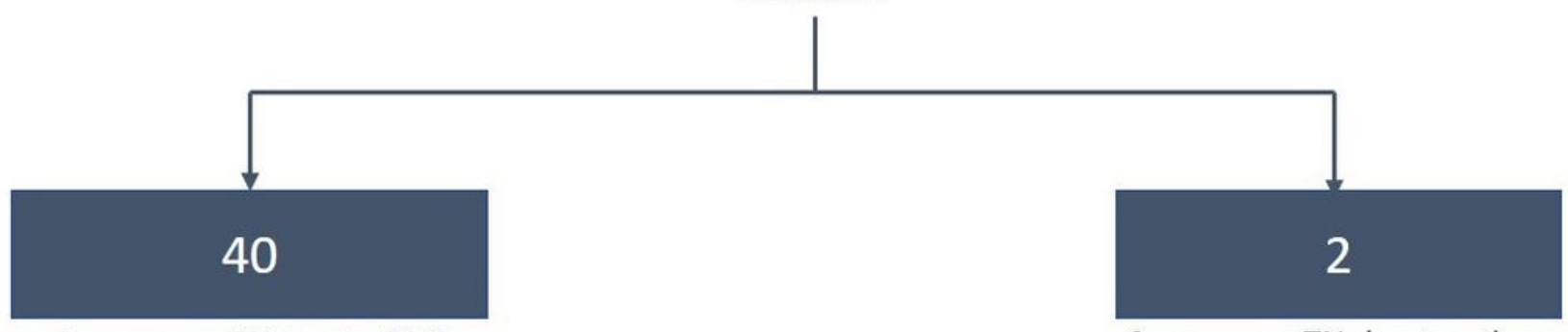

Cases were TN due to NVC

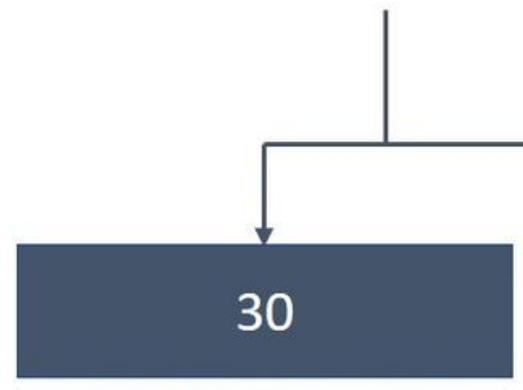

Cases were TN due to other than NVC

Cases NVC over medial aspect of Trigeminal Nerve

Cases NVC over lateral aspect of Trigeminal Nerve

\section{Figure 1}

Flow chart showing total number of cases included and excluded in our study. 


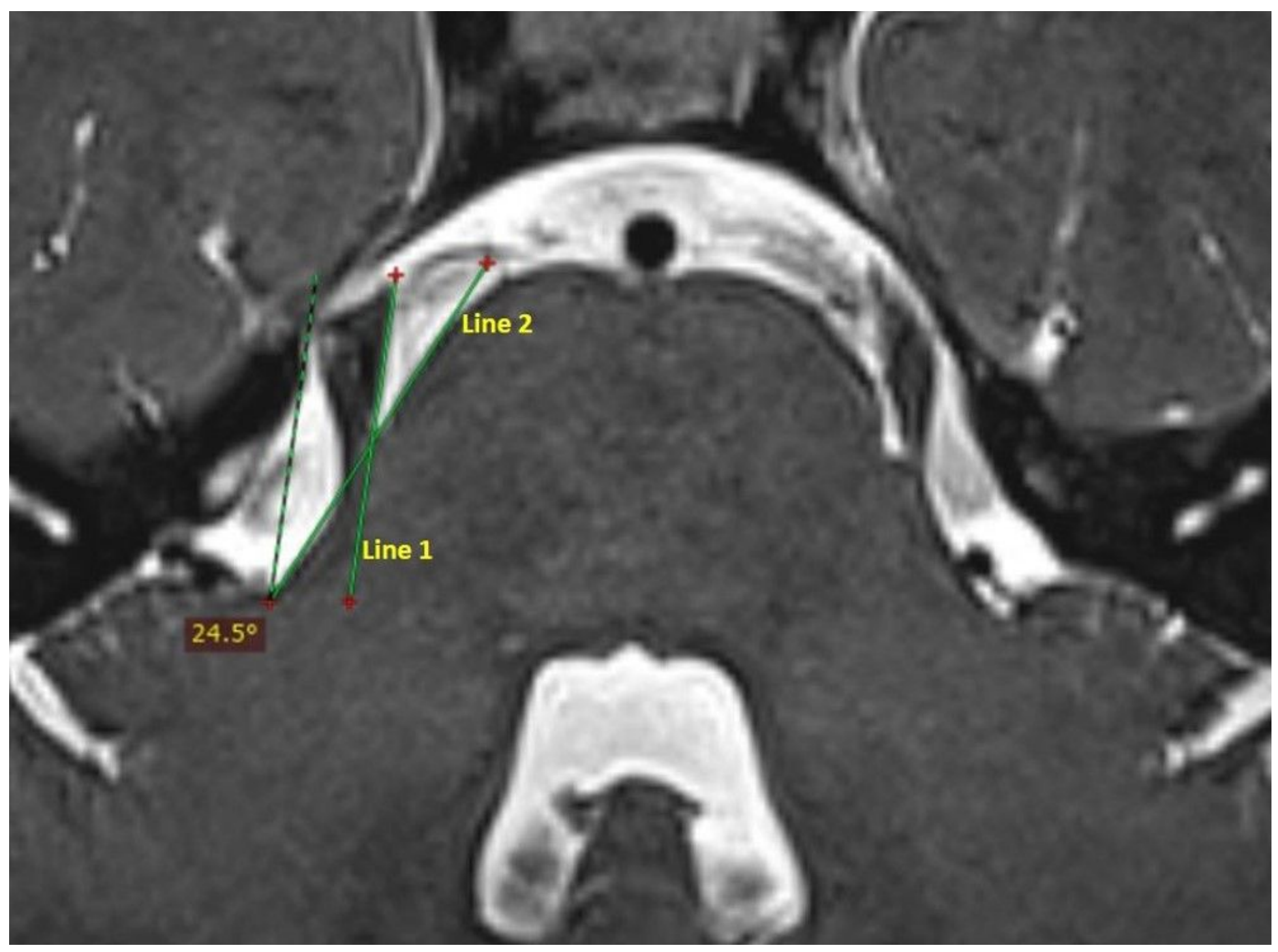

Figure 2

T2 3D space- axial sections of MRI brain shows Two-line Cobb angle method for calculation of trigeminopontine angle. 


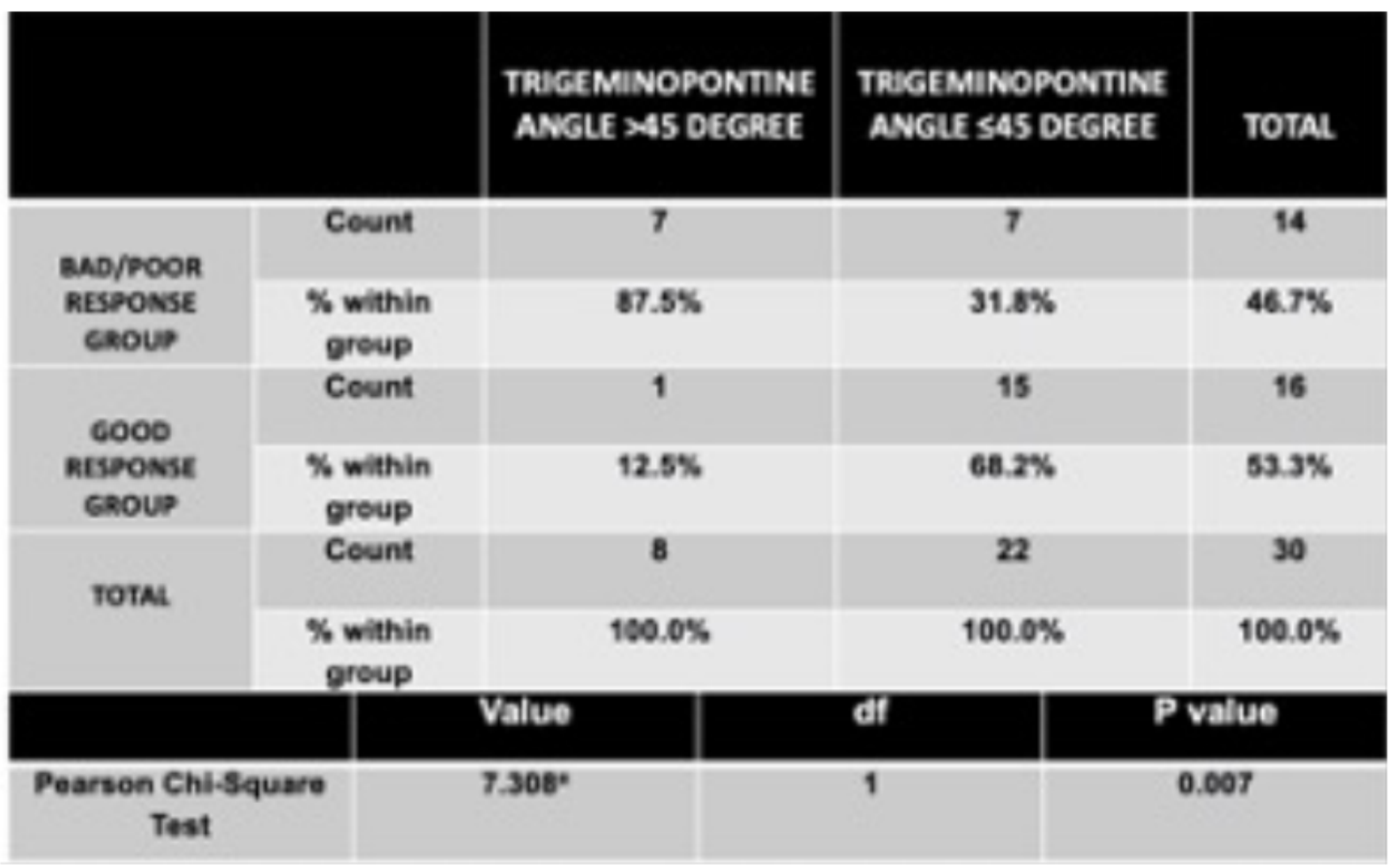

\section{Figure 3}

Statistical Analytic table showing comparison data between patients with post treatment pain relief good response and poor /bad response groups with trigeminopontine angle threshold of 45 degree. Pearson Chi-Square test shown that statistical significance difference with p-valve of 0.007 . 

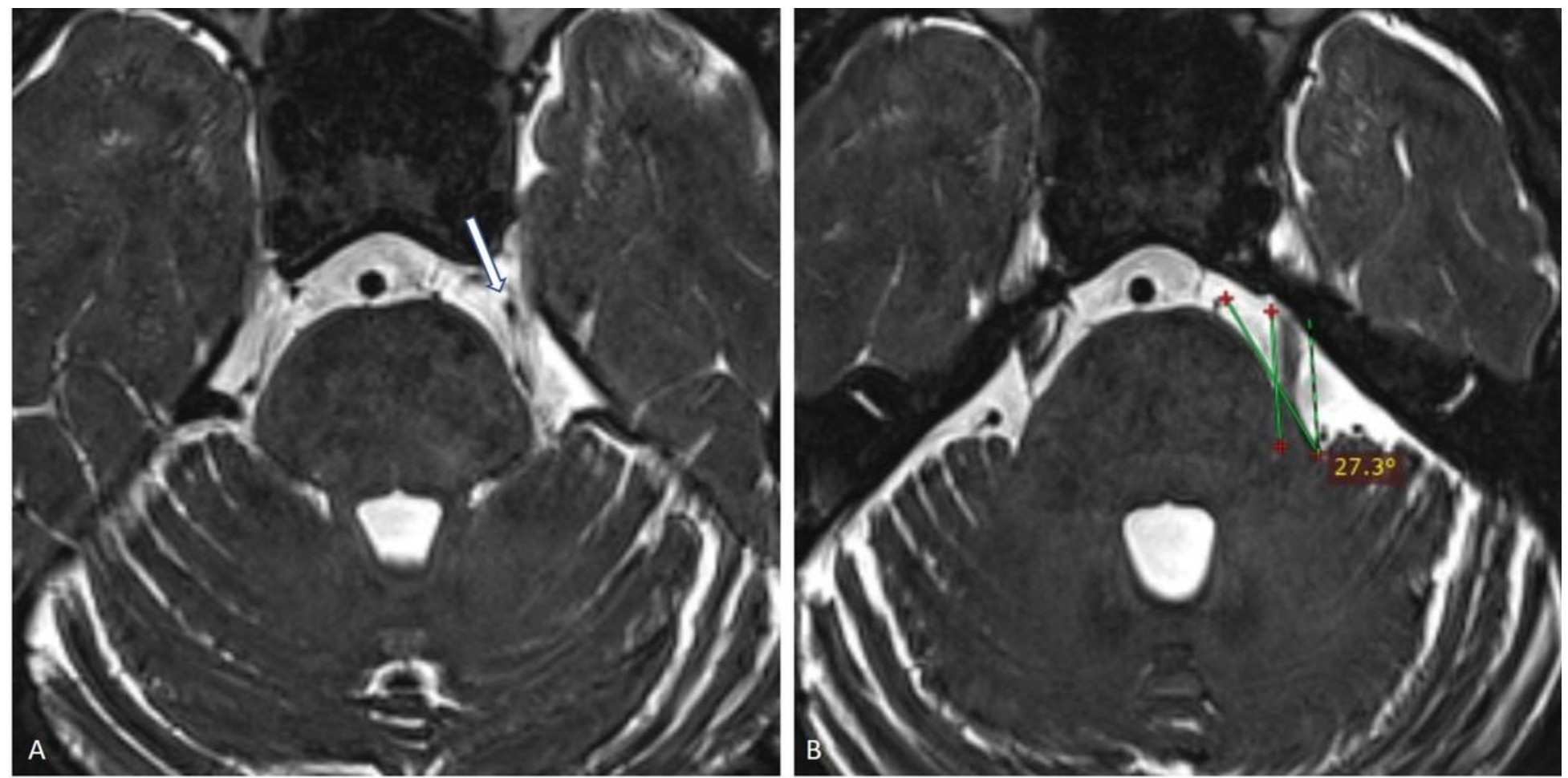

Figure 4

46 years female came with complaint of electric shock like sensation on left side of face. A) Vascular loop seen compression over medial aspect of left trigeminal nerve (arrow). B) Shows left trigeminopontine angle 27.30. Pre-treatment pain scale-7, post treatment scale-2 and post treatment pain reduction scale- 5 (71.4\%).

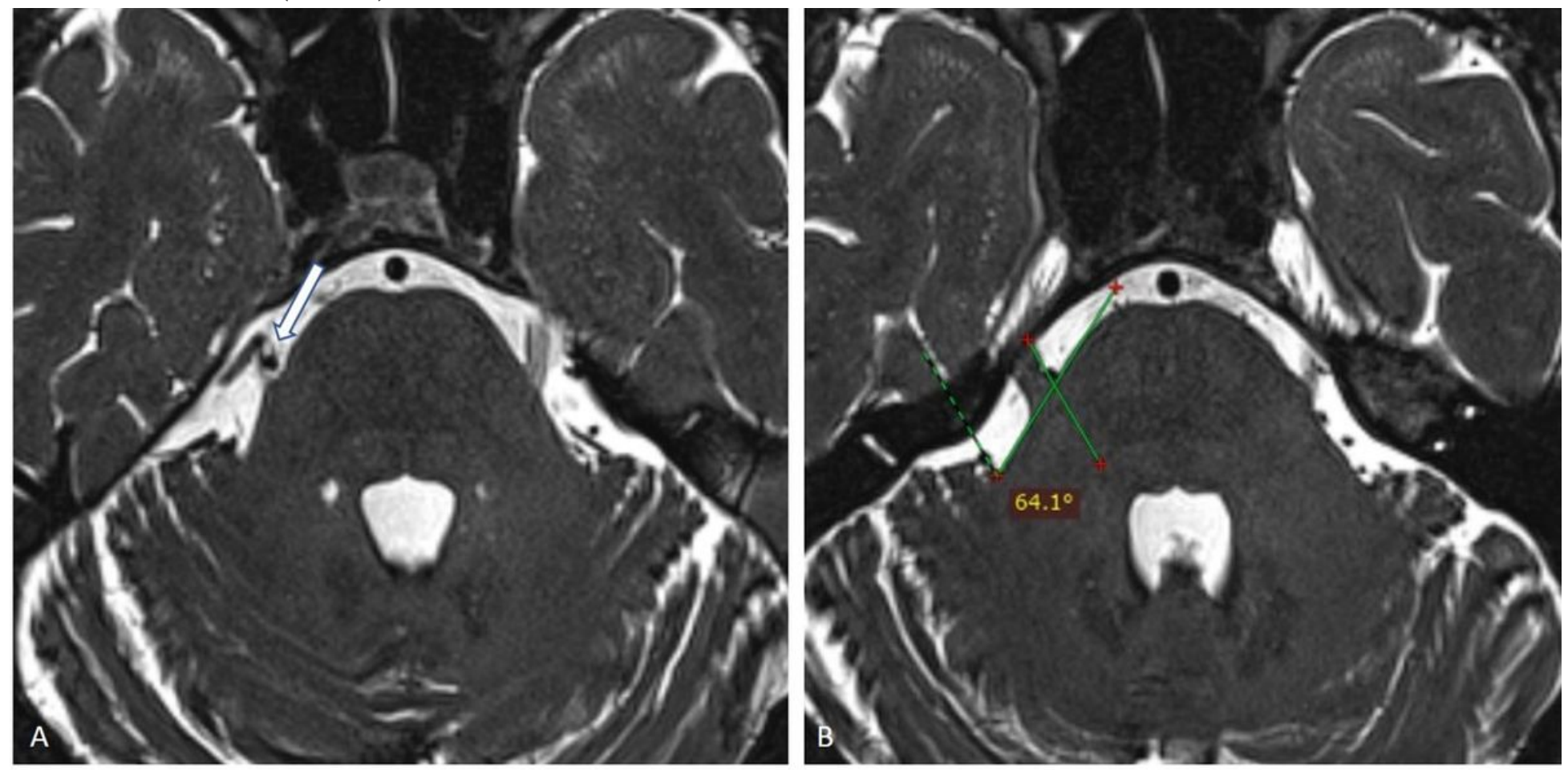

Figure 5 
39 years male came with complaint of electric shock like sensation on right side of face, very severe in intensity even with blow of air, inability to speak. A) Vascular loop seen compression over medial aspect of right trigeminal nerve (arrow). B) Shows right trigeminopontine angle 64.10. Pre-treatment pain scale-6, post treatment scale-4 and post treatment pain reduction scale- 2 (33.3\%). 